\section{Cahiers de Narratologie}

Analyse et théorie narratives

17 | 2009

Stéréotype et narration littéraire

\title{
Les proverbes dans "El ingenioso hidalgo" don Quijote de La Mancha : des stéréotypes linguistiques et culturels révélateurs de la complexité du message cervantin
}

\section{Sonia Fournet-Perot}

\section{(2) OpenEdition}

\section{Journals}

Édition électronique

URL : http://journals.openedition.org/narratologie/1288

DOI : 10.4000/narratologie. 1288

ISSN : 1765-307X

Éditeur

LIRCES

Référence électronique

Sonia Fournet-Perot, "Les proverbes dans "El ingenioso hidalgo" don Quijote de La Mancha : des stéréotypes linguistiques et culturels révélateurs de la complexité du message cervantin », Cahiers de Narratologie [En ligne], 17 | 2009, mis en ligne le 22 décembre 2009, consulté le 20 avril 2019. URL : http://journals.openedition.org/narratologie/1288; DOI : 10.4000/narratologie.1288

Ce document a été généré automatiquement le 20 avril 2019

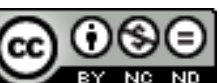

Cahiers de Narratologie - Analyse et théorie narratives est mis à disposition selon les termes de la licence Creative Commons Attribution - Pas d'Utilisation Commerciale - Pas de Modification 4.0 International. 
Les proverbes dans "El ingenioso hidalgo" don Quijote de La Mancha : des stéréotypes linguistiques et culturels révélateurs de la complexité du message cervantin

\author{
Sonia Fournet-Perot
}

1 Les séquences figées d'une langue, tels les proverbes ${ }^{1}$, sont communément regroupées sous l'appellation de « langage stéréotypique ». Dans la mesure où ces énoncés expriment une norme universelle, nous pensons qu'il est envisageable de les considérer comme des stéréotypes culturels. Afin de délimiter au mieux le champ d'application de ce que l'on entend par "langage stéréotypique », il semble nécessaire, dans un premier temps, de définir la notion de stéréotype. Le terme "stéréotype " trouve son origine dans la typographie. Le Littré le définit comme suit : « terme d'imprimerie. Il se dit des ouvrages imprimés avec des pages ou planches dont les caractères ne sont pas mobiles, et que l'on conserve pour de nouveaux tirages ${ }^{2} »$. Ce sens technique relatif à l'imprimerie s'est progressivement étendu et a recouvert le sens de fixité : «qui ne se modifie point, qui reste toujours le même ». Le stéréotype, au sens de schéma ou de formule figée n'apparaît qu'au XXe siècle et devient, dans les années vingt, un centre d'intérêt pour les sciences sociales. C'est l'Américain Walter Lippman qui a introduit pour la première fois en 1922 la notion de stéréotype dans son ouvrage intitulé Opinion publique. Par ce terme passé du jargon technique de l'imprimerie au langage courant, il désigne les représentations toutes faites, les schémas culturels préexistants, grâce auxquels chaque individu filtre la réalité. En effet, sans ces représentations, il serait impossible de comprendre le réel, de le catégoriser ou encore d'agir sur lui : comment pourrions-nous examiner chaque être, chaque objet en détail et dans sa spécificité propre sans le relier à un type ou à une généralité ? Une telle tentative n'aurait, selon Lippman, aucune chance de réussite. 
2 Cette réflexion a donné lieu à bon nombre de travaux ayant essentiellement trait à la psychologie sociale; ils soulignent le fait que le stéréotype, relevant d'un processus de catégorisation et de généralisation, simplifie et élague le réel et peut donner naissance à des visions schématiques, voire déformées de l'autre, favorisant l'apparition de préjugés. Cependant, comme l'affirment Ruth Amossy et Anne Herschberg Pierrot, si le stéréotype a tout d'abord été déprécié en raison de ces travers (trop simpliste, enclin à présenter une image erronée de la réalité) dans les tentatives de définition dont il a fait l'objet ${ }^{3}$, il a ensuite, à partir des années cinquante, connu une réhabilitation due au fait que l'homme a besoin de se rapporter à des modèles préexistants pour pouvoir comprendre le monde et régler ses conduites. Les Sciences du langage, et plus précisément la sémantique et la pragmatique intégrée, voient quant à elles dans le stéréotype une représentation simplifiée associée à un mot. Aujourd'hui, le terme de stéréotype continue à "désigner une image collective figée ${ }^{4} »$. Le langage stéréotypique représente ainsi l'ensemble des structures de la langue mettant en avant le savoir partagé et la connaissance du monde d'une société linguistique.

3 Quelles raisons nous autorisent à penser que les proverbes sont des stéréotypes? Ces énoncés constituent la sagesse des nations ou sagesse populaire, c'est-à-dire, en termes moins elliptiques, la morale d'un peuple précis à une époque donnée. L'accent est mis sur la volonté de transmettre une sagesse. La matière proverbiale représente donc, pour reprendre les mots de Henri Meschonnic, « une tentative empirique de mettre le monde en ordre ${ }^{5}$ ». Nous rejoignons ici la fonction fondamentale du stéréotype qui est de désigner une image collective figée grâce à laquelle chaque individu a la possibilité de régler ses actions. Citons, par exemple, afin d'illustrer notre propos, les énoncés «Quien puede y no quiere, cuando querrá no podrá » [Qui peut et ne veut pas, quand il voudra il ne pourra pas] ou "El hábito no hace al monje » [L'habit ne fait pas le moine]. Ces séquences présentent une sagesse qui tente de schématiser et de catégoriser le monde qui nous entoure. Les proverbes peuvent donc être considérés comme étant ou véhiculant des stéréotypes culturels et en possèdent une caractéristique essentielle : le figement, qui se traduit, au niveau formel, par l'existence d'un moule linguistique, à la fois rhétorique et syntaxique, spécifique.

4 Notre but est ici de tenter de démontrer que ces stéréotypes, à la fois linguistiques et culturels, ne sont pas de simples ornements mais ont pour fonction de servir, en discours, la stratégie énonciative de l'œuvre littéraire au sein de laquelle ils sont insérés. Nous nous intéresserons en particulier au cas de l'œuvre majeure de Cervantès, le Don Quijote de la Mancha ${ }^{6}$. L'objectif affiché du Quichotte serait de rendre détestable au public les histoires imaginaires et absurdes des romans de chevalerie ${ }^{7}$. En effet, le narrateur nous présente les aventures de don Quichotte et de son écuyer Sancho. Le premier, rendu fou par la lecture excessive de romans de chevalerie, se prend pour un chevalier errant et vit dans un monde imaginaire calqué sur celui de la littérature chevaleresque. Archétype de l'idéalisme, il incarne la quête utopique d'une société où seraient respectées les vertus cardinales et théologales. Son écuyer, le naïf mais si pragmatique Sancho Panza, suit son maître dans ses pérégrinations, tout en lui rappelant régulièrement les contraintes du réel. Un tel écart entre rêve et "réalité », entre spiritualisme et matérialisme, engendre des situations où prédominent l'ironie, l'humour, voire l'absurde, comme en témoigne l'analyse de l' "applicabilité $»^{8}$ - adéquation d'un énoncé à un contexte particulier (ici celui de l'œuvre littéraire analysée) - des proverbes, sujette elle-aussi à ce décalage constant. L'analyse de cette matière proverbiale va contribuer à l'émergence d'un autre 
niveau de lecture dont l'effet de sens global serait l'ambiguïté, éliminant ainsi toute interprétation monolithique. La réception (effet produit et sens) d'une œuvre consistant en une appropriation active qui en modifie la valeur et le sens au fil du temps, tout ouvrage s'inscrit dans une historicité de réception ${ }^{9}$ ce qui, ici, contribue à la multiplication des interprétations envisageables. L'usage des énoncés proverbiaux serait ainsi susceptible de varier selon le point de vue adopté et cette polyvalence pourrait traduire la complexité du Janus à double face que semble être le Quichotte.

10

5 Les énoncés proverbiaux semblent réservés aux deux personnages principaux de l'œuvre : don Quichotte, qui prononce $31.4 \%$ des occurrences, et Sancho Panza, $30 \%$. Vient ensuite le narrateur avec $10 \%$ des énoncés relevés. Les autres énonciateurs se limitent, quant à eux, à un ou deux proverbes. Le Chevalier à la Triste Figure et son écuyer sont non seulement les principaux énonciateurs de proverbes mais chacun est aussi l'interlocuteur privilégié de l'autre : don Quichotte adresse $64 \%$ environ des séquences proverbiales qu'il emploie à Sancho et ce dernier $81 \%$ à son maître.

6 Certains énoncés proverbiaux ne sont pas applicables à leurs énonciateurs qui, de par leur caractère ou leur façon d'agir, s'avèrent être en confrontation avec la sagesse véhiculée. Cette caractéristique s'appliquerait plus particulièrement à don Quichotte et à Sancho. Le premier a sombré dans la folie et croit vivre au temps des chevaliers dont il défend les principes tout droit sortis de ses livres de fiction. En proie à une perception faussée de la "réalité ", il considère les choses et les individus qui croisent son chemin sous une apparence idéale, à l'image des topiques du roman chevaleresque dont il est imprégné : il s'imagine, par exemple, que la bassine du barbier est un heaume légendaire en piteux état; une simple auberge devient un palais, l'aubergiste, un grand seigneur... Sa conception du bien et du mal est également altérée: lorsqu'il croise un groupe de prisonniers que l'on conduit aux galères, il les libère au nom du principe chevaleresque qui veut que l'on vienne en aide aux plus faibles... Sancho n'est pas atteint de folie mais est naïf et intéressé, ce qui le conduit à accepter tout ce que lui dit son maître, que cela ait trait aux magiciens, dragons ou autres chimères. Bien qu'il ait pu constater, à maintes reprises, que don Quichotte perdait son jugement, il continue de le servir avec dévouement dans l'attente d'une rétribution fabuleuse. Comment ne pas sourire lorsque nous, lecteurs modernes, constatons qu'ils prononcent à eux deux la majorité des proverbes de l'œuvre alors que la matière proverbiale est assimilée à la sagesse ? Nous pourrions interpréter cela comme de l'ironie : l'emploi des proverbes - non applicables à leurs énonciateurs - révèlerait une critique de l'auteur envers ses personnages. Mais il pourrait également s'agir d'une invitation que Cervantès adresse à son public à jeter un regard sur ces stéréotypes linguistico-culturels particuliers. En effet, don Quichotte affirme, p. 252 :

Paréceme, Sancho, que no hay refrán que no sea verdadero, porque todos son sentencias sacadas de la mesma experiencia, madre de las ciencias todas (...). [Il me semble, Sancho, qu'il n'est pas de proverbe qui ne soit vrai, parce qu'ils sont tous des sentences issues de l'expérience même, mère de toutes les sciences $\left.{ }^{11}(. .).\right]$.

7 De même qu'il croit aux idéaux chevaleresques décrits dans ses livres, don Quichotte croit à la sagesse proverbiale. Les proverbes étant prononcés en majorité par un fou et un naïf, nous pourrions en déduire qu'il faut être fou ou naïf pour croire en la sagesse des énoncés proverbiaux et tenter de la mettre en pratique. Cette hypothèse se verrait confirmée par 
la présence redondante, dans le corpus en question, de proverbes contraires, dont voici un exemple éloquent :

- No te dé pena eso, señora mía - respondió Leonela - ; que no está la monta ni es causa para menguar la estimación darse lo que se da presto, si, en efecto, lo que se da es bueno, y ello por sí digno de estimarse. $Y$ aun suele decirse que el que luego da, da dos veces.

- También se suele decir - dijo Camila -, que lo que cuesta poco se estima en menos.

- No corre por ti esa razón - respondió Leonela -, porque el amor, según he oído decir, unas veces vuela y otras anda (...). (pp. 423-424)

[- Que cela ne te cause point de peine, ma dame - répondit Leonela - ; car cela n'a point d'importance et ne fait point diminuer l'estime que l'on a de nous que de donner prestement ce que l'on donne, si, en effet, ce que l'on donne est bon et digne d'estime. On a même coutume de dire que celui qui donne vite, donne deux fois. - On a aussi coutume de dire - dit Camila -, que ce qui coûte peu est peu estimé.

- Ce n'est pas à toi que s'adresse cet argument - répondit Leonela-, parce que l'amour, d'après ce que j'ai entendu dire, certaines fois vole et d'autres marche (...).]

Les deux premiers énoncés impliquent une conclusion factuelle (impliquant une demande d'action) opposée : « on doit donner vite » vs « on ne doit pas donner vite ». Le troisième implique qu'en ce qui concerne le cas bien particulier de l'amour, il n'est pas de règle précise. Comment, donc, organiser sa vie autour de séquences proverbiales qui sont susceptibles de se contredire? Cela paraît contraire à la raison. Pourtant, c'est ce que tentent de réaliser nos deux héros. L'auteur du Quichotte semble dire à son lecteur qu'il ne faut pas croire à tout ce que relatent les romans de chevalerie et ne pas chercher à en appliquer les valeurs à la "réalité »; il pourrait également lui signifier, par le biais de l'emploi des proverbes, que l'on ne doit pas croire tout ce qu'affirme la sagesse populaire, comme le fait notre chevalier errant, ni vouloir l'appliquer au monde dans lequel on vit, comme s'y emploie l'écuyer qui n'a de cesse, tout au long de l'œuvre, d'enchaîner les proverbes. L'usage que l'auteur fait des séquences proverbiales conforterait donc la volonté énonciative de l'œuvre.

Il nous semble cependant que cette analyse n'est que superficielle. Nombre de critiques de Cervantès ont prétendu que le message de $E l$ ingenioso hidalgo était beaucoup plus complexe ${ }^{12}$. Nous tendons à partager cette interprétation dans et à travers le temps et l'analyse de l'utilisation de la matière proverbiale semble appuyer ces hypothèses. Un épisode, au début de l'œuvre, sème tout d'abord le doute quant au véritable message que désirait délivrer Cervantès : au cours de l'autodafé organisé par le barbier et le curé afin de libérer leur ami de l'emprise des romans de chevalerie, ils en épargnent néanmoins quelques uns: l'Amadís de Gaula, le Palmerín de Ingalaterra et la Historia del famoso caballero Tirante el Blanco. Les autres sont condamnés au bûcher pour leur manque de réalisme. Cette remarque nuancerait déjà nos précédents propos. Par ailleurs, le roman de chevalerie tendait à présenter la vision d'un monde souhaité : non pas la « réalité », mais l'image idéale que l'on aimerait qu'elle recouvre. Don Quichotte vit et agit dans le respect de valeurs utopistes qui altèrent sa perception de la «réalité » et qui provoquent l'incompréhension de ses contemporains, lesquels ne voient en lui que folie. Mais est-on vraiment fou parce que l'on veut croire en un monde meilleur et que l'on pense être investi de la mission de faire respecter l'ordre et la justice, la tempérance, la sagesse et l'amour de Dieu? Est-on vraiment fou parce qu'on se nourrit de littérature? Rêveur, peut-être ; fou, nous ne le pensons pas. Don Quichotte agit de façon absurde mais sa quête 
(améliorer le monde) ne l'est pas ; c'est le monde dans lequel il vit, ce monde qui sombre dans la décadence, qui peut être considéré comme contraire à la raison ${ }^{13}$. Peut-être que cet hidalgo de la Manche est en réalité le moins fou de tous ; peut-être que notre crédule Sancho, rendu pur par sa naïveté, perçoit la sagesse de la démarche de son maître et le suit pour cela fidèlement. Peut-être qu'un possible message de l'œuvre serait : l'utopie n'est pas folie ; c'est de laisser le monde tel qu'il est, sans chercher à l'améliorer, qui serait folie. Si nous adoptons cette interprétation, nous invalidons la décrédibilisation de l'application de la sagesse proverbiale en même temps que la folie de don Quichotte. Les proverbes porteraient tous en eux une vérité, un bon sens que l'on ferait mieux d'écouter. Deux proverbes contraires peuvent exister sans que cela remette en cause leur véracité : tout est fonction de la situation d'énonciation à laquelle ils sont incorporés. De la même façon qu'il faudrait savoir déceler la sagesse dans la folie de don Quichotte, le bon sens dans la naïveté de Sancho, il faudrait voir la véritable sagesse de la matière proverbiale dans ses contradictions.

Que nous considérions que Cervantès ait voulu critiquer la matière proverbiale ou, au contraire, mettre sa sagesse en évidence en choisissant don Quichotte ou Sancho comme énonciateurs conditionne totalement notre perception de l'emploi ironique des proverbes. Il y aurait donc deux degrés de lecture possibles:

- un premier degré, superficiel, (critique du roman de chevalerie et de ceux qui ne distinguent pas la fiction de la « réalité »; don Quichotte est un fou, Sancho un paysan nigaud), qui impliquerait, lors du choix des énonciateurs de proverbes, une interprétation ironique.

- un second degré, plus profond, (critique du monde réel où règnent injustice, intolérance, immoralité, égoïsme..., et présentation de don Quichotte comme incarnation de la sagesse et de la bonté) qui induirait une interprétation sérieuse.

11 Qu'en est-il de l'ironie découlant de la sélection d'une situation d'énonciation particulière?

Concernant un cas isolé, la volonté ironique de l'auteur et du narrateur parait évidente :

Creedme, fermosa señora, que os podéis llamar venturosa por haber alojado en este vuestro castillo a mi persona, que es tal, que si yo no la alabo es por lo que suele decirse que la alabanza propria envilece ; (p. 200)

[Croyez-moi, belle dame, vous pouvez vous dire heureuse d'avoir accueilli dans votre château ma personne, qui est telle, que si je n'en fais pas l'éloge, c'est parce que l'on a coutume de dire que l'éloge de sa propre personne avilit ;]

La situation d'énonciation immédiate n'autorise pas l'emploi du proverbe : don Quichotte recourt à un proverbe induisant qu'il ne faut pas se vanter alors qu'il vient d'affirmer à son hôtesse qu'elle peut s'estimer chanceuse de recevoir une personne telle que lui sous son toit. Le gentilhomme ne se rend pas compte de la contradiction de ses propos. En revanche, nous pourrions penser que le narrateur et l'auteur en sont conscients et désirent que le lecteur perçoive la non-applicabilité du proverbe à la situation. Nous serions donc bien en présence d'ironie exprimée à l'encontre du personnage. Cependant, il ne s'agit pas d'une ironie cinglante mais source d'une moquerie bon enfant plutôt qu'acerbe. Cet état de fait pourrait trouver une explication dans le caractère même de don Quichotte : en décalage avec la « réalité », le héros n'en demeure pas moins sincère et épris de justice ce qui pourrait rendre le lecteur plus indulgent devant les absurdités qu'il peut dire ou commettre. D'autres emplois proverbiaux sont plus problématiques. C'est le cas lorsque don Quichotte croise le chemin de prisonniers que l'on emmène aux galères. Considérant qu'il s'agit d'opprimés, notre héros décide de les libérer de leurs chaînes. 
Toutefois, il veut tenter d'éviter toute violence envers les gardes et choisit donc d'exposer calmement sa requête en se justifiant par l'énoncé proverbial : «lo que se puede hacer por bien no se haga por mal » [ce que l'on peut obtenir de bon gré, il ne faut pas l'obtenir par la force] (p. 273).

La non-applicabilité réside ici dans le fait que ce que veut faire le pseudo chevalier errant, c'est redonner la liberté à des voleurs, escrocs et autres criminels. Un proverbe, de par la sagesse dont il est le représentant, ne peut en aucun cas être employé pour justifier pareil comportement. Le lecteur sourit mais il ne le fait pas avec malice. Il nous semble que le statut hors du commun de don Quichotte, en totale déconnexion avec la "réalité", entrave l'installation d'une exégèse ironique acerbe. Cette perception indulgente se verrait confirmée par la seconde thèse défendue quant au possible message de l'œuvre selon laquelle notre hidalgo serait un sage vivant au milieu d'un monde en pleine dégénérescence. Cette possibilité d'interprétation rend difficile l'analyse d'exemples comme celui que nous venons d'examiner : si les agissements de don Quichotte visent à une amélioration de la justice, à une quête des grandes vertus, alors l'emploi du proverbe appartient à un discours sérieux. Comme précédemment, coexistent, à des degrés différents, deux lectures possibles. Le lecteur perçoit chacune d'elles: la première, tendrement railleuse, l'incite à sourire, la seconde, plus intellectuelle, le pousse à réfléchir sur la réalité de l'univers dans lequel il vit. L'usage de proverbes dans des situations avec lesquelles ils sont en apparente contradiction est un moyen de détecter cette double lecture dans la mesure où il incite à se poser la question de l'adéquation de leur emploi. Plutôt que l'ironie, nous pensons que c'est l'humour qui est souvent recherché dans l'utilisation des proverbes par Cervantès.

Nous avons, jusqu'à présent, restreint la non-applicabilité proverbiale au domaine de l'ironie. Or, il est des cas où un proverbe non-applicable n'a pas pour origine une volonté critique dissimulée mais une volonté humoristique ouverte. Autrement dit, on ne cherche pas à faire entendre le contraire (ou quelque chose de différent) de ce que l'on dit ou montre, mais on désire simplement mettre en évidence le caractère grotesque de la situation. L'effet humoristique d'une non-applicabilité proverbiale pourrait tenir au fait que l'énonciateur concerné est en total décalage avec la « réalité » ou ce qui est reconnu comme telle.

- Non-applicabilité situationnelle ayant pour origine une contrariété

Voici un exemple à l'origine de cette réflexion : durant la nuit, la fille de l'aubergiste et sa servante Maritornes veulent se jouer de don Quichotte; elles lui demandent de s'approcher de la lucarne du grenier depuis laquelle elles l'observent, montant la garde. Le gentilhomme est persuadé qu'il s'agit d'une noble dame, accompagnée de sa duègne, qui s'est éprise de sa personne; il s'avance donc vers la lucarne en précisant à la dame que son cœur appartient à Dulcinea del Toboso, mais qu'il est prêt à lui rendre service. Maritornes lui rétorque que sa maîtresse a simplement besoin de l'une de ses mains. Le chevalier errant s'empresse de répondre à cette requête et, se mettant debout sur la selle de Rocinante pour atteindre la lucarne, tend la main à celle qu'il suppose être une noble dame. Maritornes lui attache alors le poignet au verrou de la porte du grenier. Don Quichotte, sentant la corde lui serrer le poignet s'étonne :

Más parece que vuestra merced me ralla que no que me regala la mano; no la tratéis tan mal, pues ella no tiene la culpa del mal que mi voluntad os hace, ni es 
bien que en tan poca parte venguéis el todo de vuestro enojo. Mirad que quien quiere bien no se venga tan mal. (p. 528)

[Il semble que votre grâce me râpe la main plutôt qu'elle ne me la flatte; ne la traitez pas si mal, car elle n'est pas coupable du mal que vous font mes sentiments, et ce n'est pas bien non plus que vous vengiez sur une si petite partie de ma personne toute votre colère. Notez que qui aime bien ne se venge pas si méchamment.]

17 Le proverbe employé sous-entend que la fille de l'aubergiste « aime bien » don Quichotte alors que c'est loin d'être le cas. La séquence proverbiale est non-applicable à la situation d'énonciation. Il ne s'agit pourtant pas d'ironie. Tout d'abord, parce que don Quichotte voit les choses de manière utopique, distinctes de ce qu'elles sont en vérité ; ensuite, parce que cette non-applicabilité ne déclenche pas chez le lecteur une réflexion lui permettant d'aboutir à une critique quelconque du personnage mais le rire. Un individu possédant toute sa raison ne bénéficierait pas, dans des circonstances identiques, de la même clémence. Sa perception faussée de la « réalité » donne lieu à des évènements que nous pourrions qualifier de burlesques. Par burlesque nous entendons désigner un certain type d'humour qui repose sur l'extravagance des situations ${ }^{15}$. La posture ridicule de don Quichotte et sa vision de ce qui est en train de se produire concourent à la création d'une scène burlesque. Le proverbe, représentant de la sagesse, jure avec le tableau qui nous est donné à voir et représente donc le côté sérieux du personnage dont la position est pourtant ridicule, encline à provoquer involontairement le rire. La matière proverbiale pourrait donc, en cas de non-applicabilité, être un marqueur d'humour et, plus précisément ici, de burlesque.

- Non-applicabilité situationnelle ayant pour origine une utilisation grossière du proverbe

Sancho, lui aussi, prononce un énoncé proverbial non-applicable à la situation en ce sens qu'il utilise un proverbe pour parler de choses vulgaires, grossières. En effet, lorsque don Quichotte s'enquiert auprès de son écuyer du parfum de sa dulcinée, celui-ci lui répond :

- Lo que sé decir - dijo Sancho - es que sentí un olorcito algo hombruno ; y debía de ser que ella, con el mucho ejercicio, estaba sudada y algo correosa.

- No sería eso - respondió don Quijote - ; sino que tú debías de estar romadizado, o te debiste de oler a ti mismo; porque yo sé bien a lo que huele aquella rosa entre espinas, aquel lirio del campo, aquel ámbar desleído.

- Todo puede ser - respondió Sancho - ; que muchas veces sale de mí aquel olor que entonces me pareció que salía de su merced de la señora Dulcinea; pero no hay de qué maravillarse, que un diablo parece a otro. (p. 384)

[- Ce que je puis dire - dit Sancho - c'est que j'ai senti une petite odeur un peu hommasse ; et ce devait être qu'à force d'exercice, elle était en sueur et un peu sale.

- Ça ne pouvait pas être ça -< répondit don Quichotte - ; mais toi, tu devais avoir un rhume de cerveau, ou tu as du sentir ta propre odeur ; parce que je sais bien, moi, ce que sent cette rose parmi les épines, ce lys des champs, cet ambre délayé.<br />Tout est possible - répondit Sancho - ; car, très souvent, sort de moi cette odeur qui m'a alors semblé sortir de sa grâce dame Dulcinea; mais il n'y a pas de quoi s'étonner, car un diable ressemble à un autre.]

19 Le message du proverbe est ici réduit à souligner la similitude des odeurs corporelles. L'énoncé gnomique est non-applicable à la situation, non parce qu'il existe une contrariété (des sueurs peuvent effectivement être aussi nauséabondes les unes que les autres...), mais parce que la situation d'énonciation implique un emploi vulgaire de la séquence proverbiale qui n'est pas compatible avec la sagesse qu'elle véhicule. Le proverbe est en décalage avec le thème (la sueur) auquel il se trouve appliqué : le spirituel est rabaissé au niveau physique. En employant la matière proverbiale dans un 
contextegrossier, Sancho crée le contraste entre noblesse (proverbe) et familiarité / bassesse (sujet de la conversation) qui permet la naissance du burlesque. Nous pourrions également considérer que ce proverbe contribue indirectement - à travers l'un de ses éléments - à l'élaboration d'un jeu de mots construit autour de la polysémie des termes «olor» [odeur / réputation] et « diablo" [personne quelconque (sens premier dans la séquence étudiée) / diable, personne mauvaise]. En effet, l'association de ces deux substantifs pourrait constituer une référence antiphrastique à la locution «olor de santidad» [odeur de sainteté]. Cette relation impliquant un terme de l'énoncé proverbial induirait une critique involontaire de la part de Sancho quant à sa propre réputation et quant à celle de Dulcinea, ce qui pourrait amplifier l'effet humoristique déclenché par la non-applicabilité ${ }^{16}$. La trivialité est également susceptible d'apparaître, comme nous venons de l'entrevoir, via une bi-applicabilité du proverbe.

\section{Quichotte}

20 La bi-applicabilité a trait à l'utilisation de jeux de mots. Ceux-ci peuvent être simplement humoristiques ou tomber dans la vulgarité.

- Jeux de mots humoristiques grossiers : la mise en place du burlesque

21 L'exemple le plus explicite se situe p. 246 de l'œuvre étudiée : Sancho, terrorisé au milieu de la nuit par un vacarme d'origine inconnue, se tient tout contre son maître. Lui prend l'envie impérieuse de satisfaire un besoin naturel. Sa peur l'empêche de bouger et il évacue ses matières fécales sur place. Si la chose s'avère, au soulagement de Sancho, très peu bruyante, elle se révèle, en revanche, fort odorante, ce qui ne manque pas d'incommoder le nez délicat de notre gentilhomme :

- Retírate tres o cuatro allá, amigo - dijo don Quijote, todo esto sin quitarse los dedos de las narices -, y desde aquí adelante ten más cuenta con tu persona y con lo que debes a la mía; que la mucha conversación que tengo contigo ha engendrado este menosprecio.

- Apostaré - réplicó Sancho - que piensa vuestra merced que yo he hecho de mi persona alguna cosa que no deba.

- Peor es meneallo, amigo Sancho - respondió don Quijote.

[- Retire-toi de trois ou quatre pas, mon ami - dit don Quichotte, sans cesser de se pincer le nez avec les doigts -, et dorénavant prends plus garde à ta personne et à ce que tu dois à la mienne ; le fait de beaucoup converser avec toi a engendré un tel mépris.

- Je parierai - répliqua Sancho - que votre grâce pense que j'ai fait physiquement quelque chose que je ne dois pas.

- C'est pire de le remuer, ami Sancho - répondit don Quichotte.]

«Peor es meneallo» fait référence au proverbe d'expression indirecte ${ }^{17}$ « La mierda, cuanto más se menea, más apesta » [La merde, plus on la remue, plus elle pue], soit, après démétaphorisation, "si on insiste à propos d'un fait désagréable, cela fait empirer les choses ». L'humour tient ici au fait que le proverbe est bi-applicable à la situation : que l'on considère son message figuratif ou non figuratif, l'applicabilité est possible. La métaphore employée renvoie au contexte immédiat : don Quichotte réprimande Sancho concernant son comportement irrespectueux puisqu'il s'est permis de déféquer à côté de lui (sens transparent de « mierda»). Le serviteur tente de nier les faits, ce qui conduit le gentilhomme à lui demander de ne pas insister à propos d'un évènement déjà désagréable (sens opaque de «mierda»). Le double sens du terme grossier sous-entendu renforce le contraste entre le message sérieux du proverbe (même grossier) et la scène scatologique vécue par les deux protagonistes et permet l'apparition du burlesque. Cet exemple met 
particulièrement en évidence la réflexion de l'auteur quant au choix des proverbes employés.

- Jeux de mots humoristiques non grossiers : la mise en évidence de l'être et de la vérité rester fidèle à l'amour qu'il ressent pour sa Dulcinea del Toboso, alors qu'il ne sait pas si elle consentira à l'épouser, plutôt que de convoler avec la noble Dorotea qu'il pense être la princesse Micomicona. Le proverbe employé par l'écuyer indique que ce qui est moindre mais sûr est préférable à ce qui est de plus grande valeur mais incertain. Si le terme "pájaro" [petit oiseau] renvoie analogiquement à la jeune et belle Dorotea, «buitre » [vautour] fait référence à Dulcinea, c'est-à-dire à la paysanne hommasse et inculte en qui don Quichotte voit une dame à la beauté sans pareille. Le substantif "buitre » [vautour], en espagnol comme en français, désigne un grand oiseau de proie aux ailes vastes, au bec crochu, à la tête et au cou dénudés ou une personne rapace, dure. L'analogie n'est pas avantageuse pour la mie de notre chevalier errant que l'on considère le sens transparent (un vautour est un oiseau de proie) ou au moins deux des sens opaques de ce terme polysémique (une personne rapace, une personne laide). La biapplicabilité permet de révéler la véritable nature du personnage. L'humour impliqué par des analogies peu flatteuses lors de l'utilisation de séquences proverbiales pourrait rétablir la vraie personnalité des individus qui gravitent autour du gentilhomme de la Manche et briser la vision idéalisée que ce dernier nous en proposait. Le fait qu'il ne perçoive pas le double sens de ses propos pourrait être une des manières de mettre en évidence cette rêverie qui le fait vivre dans un monde imaginaire et utopique.

- La bi-applicabilité d'un terme conduit à un quiproquo

Par « quiproquo ", nous entendons " méprise, malentendu faisant prendre une personne, une chose pour une autre $\aleph^{18}$. Ce phénomène se produit lorsque don Quichotte converse avec les galériens et les questionne sur les raisons de leur condamnation, p. 267 :

- Éste, señor, va por canario, digo, por músico y cantor.

- Pues ¿ cómo ? - repitió don Quijote -. ¿ Por músicos y cantores van también a galeras?

- Sí, señor - respondió el galeote - ; que no hay peor cosa que cantar en el ansia.

- Antes he yo oído decir - dijo don Quijote - que quien canta, sus males espanta.

- Acá es el revés - dijo el galeote - ; que quien canta una vez, llora toda la vida.

- No lo entiendo - dijo don Quijote. 
[- Celui-ci, seigneur, va aux galères en tant que serin des Canaries, je veux dire, en tant que musicien et chanteur.

- Comment donc? - reprit don Quichotte -. On condamne aussi aux galères les musiciens et les chanteurs?

- Oui, seigneur - répondit le galérien - ; car il n'est pire chose que de chanter dans le tourment.

- Moi, j'ai plutôt entendu dire - dit don Quichotte - que qui chante, effraie ses malheurs.

- Ici c'est le contraire - dit le galérien - ; qui chante une fois pleure toute sa vie.

- Je n'y comprends rien - dit don Quichotte.]

Le quiproquo qui empêche don Quichotte de comprendre de quoi lui parle le galérien est dû à une bi-applicabilité du terme « cantar » qui signifie, comme le français « chanter », à la fois émettre une suite de sons modulés produits par la voix humaine et faire des aveux. Le bagnard fait bien sûr allusion au second sens de "cantar ", alors que le chevalier errant se réfère au premier. La bi-applicabilité est saisie et utilisée par le forçat mais non par don Quichotte qui reste de ce fait insensible au jeu de mots de son interlocuteur et fort sceptique en ce qui concerne la justice humaine. C'est l'incompréhension du gentilhomme qui déclenche le quiproquo et, par là même, le rire chez le lecteur, qui perçoit la pluralité sémantique des termes employés par le prisonnier. Ce quiproquo pourrait également mettre en évidence la naïveté de don Quichotte qui croit et prend au premier degré tout ce qu'on lui dit. Une telle remarque rejoindrait la critique des lecteurs de romans de chevalerie qui confondent fiction et «réalité ». Cependant, le malentendu entre le chevalier et le galérien serait également susceptible de souligner la pureté d'âme de notre héros qui ne voit pas le mal et parle et agit avec honnêteté et franchise. Cela pourrait fournir une possible réponse à son incompréhension. Nous pourrions également supposer que son incapacité à saisir un tel jeu de mots est le résultat de son côté rêveur. Il ne lui vient pas à l'esprit que « cantar " puisse signifier « avouer » car il est persuadé de vivre dans un monde où l'on respecte encore les grandes vertus cardinales et théologales. Sa vision utopiste l'empêche de songer à des comportements aussi vils et pourrait expliquer son incompréhension finale. En nous donnant à voir, par l'intermédiaire de don Quichotte, une caricature de la pureté et de la bonté, en forçant le trait puisqu'en dotant un être halluciné de ces qualités, peut-être l'auteur a-t-il souhaité renforcer le contraste entre un monde souhaité, idéal et le monde où évoluent les personnages. L'humour, par l'entremise de la bi-applicabilité d'un proverbe ou de l'un de ses composants, servirait donc les objectifs principaux de l'œuvre. Non-applicabilité et bi-applicabilité proverbiales ne sont pas les seules sources d'humour dans le Quichotte.

Il s'agit ici d'allusions à des proverbes qui, moyennant l'ajout d'un ou plusieurs termes, modifient l'énoncé d'origine de façon à permettre son applicabilité au contexte. Ces proverbes sous-entendus servent à décrire don Quichotte. Dans le cas présent, le changement par ajout a la particularité de montrer le côté ridicule, risible, du personnage. Ces transformations sont humoristiques car elles mettent en évidence l'écart entre ce qui est (l'énoncé ayant subi la modification) et ce qui devrait être (la norme, c'est-à-dire le proverbe) :

En un lugar de la Mancha, de cuyo nombre no quiero acordarme, no ha mucho tiempo que vivía un hidalgo de los de lanza en astillero, adarga antigua, rocín flaco y galgo corredor. Una olla de algo más vaca que carnero, salpicón las más noches, duelos y quebrantos los sábados, lantejas los viernes, algún palomino de añadidura los domingos, consumían las tres partes de su hacienda. (p. 69)

[Dans un village de la Mancha, dont je ne veux me rappeler le nom, vivait, il n'y a guère longtemps, un gentilhomme de ceux qui ont lance au râtelier, écu ancien, 
rosse maigre et lévrier fugueur. Une marmite de quelque chose fait avec plus de vache que de mouton, un saupiquet presque tous les soirs, des œufs frits avec du lard le samedi, des lentilles le vendredi, un pigeonneau en sus le dimanche, consumaient les trois quarts de sa fortune.] más vaca que carnero " [une marmite de quelque chose fait avec plus de vache que de mouton] à "Vaca y carnero, comer de caballero " [Vache et mouton, nourriture de chevalier]. La connaissance des proverbes s'avère indispensable, pour nous, lecteurs modernes, puisqu'elle nous apprend que don Quichotte ne suit pas exactement le «menu » propre aux nobles de rang supérieur. Ainsi, dès les premières lignes de l'œuvre, nous savons que le gentilhomme de la Mancha qui nous est décrit est hors norme. L'applicabilité du proverbe sous-entendu est forcée par l'insertion du comparatif « más... que ». Le rire est, ici aussi, l'effet recherché. En forçant l'applicabilité de la séquence proverbiale sous-entendue grâce à un rajout qui en altère le sens et la rend plaisante parce que grotesque ${ }^{20}$, on a peut-être voulu mettre en évidence la singularité du personnage ${ }^{21}$.

Les proverbes pourraient donc être employés, dans l'œuvre de Cervantès, à des fins humoristiques au moyen d'une non-applicabilité, d'une bi-applicabilité ou d'une applicabilité forcée. Si, dans certains cas, cet humour s'appuyant sur des énoncés proverbiaux s'avère burlesque et cherche donc simplement à divertir le lecteur, dans d'autres, il permet de révéler la véritable nature des personnages et de ce monde que notre héros et son fidèle écuyer arpentent en quête d'aventures. Cependant, la matière proverbiale n'est pas uniquement utilisée afin de servir des objectifs sérieux, ironiques ou humoristiques.

22

31 Nous considérons que l'emploi d'un proverbe est absurde lorsque ce dernier est hors de propos, c'est à dire non cohérent. C'est le personnage de Sancho qui multiplie les énoncés proverbiaux, parfois sans comprendre, semble-t-il, la teneur de leur message.

\section{modification du sens de l'énoncé :}

No sé esas filosofías - respondió Sancho Panza - ; mas sólo sé que tan presto tuviese yo el condado como sabría regirle; que tanta alma tengo yo como otro, y tanto cuerpo como el que más, y tan rey sería yo de mi estado como cada uno del suyo ; y siéndolo, haría lo que quisiese; y haciendo lo que quisiese, haría mi gusto; y haciendo mi gusto, estaría contento; y en estando uno contento, no tiene más que desear; y no teniendo más que desear, acabóse, y el estado venga, y a Dios y veámonos, como dijo un ciego a otro. (p. 587)

[Je n'entends rien à ces philosophies - répondit Sancho Panza - ; mais je sais bien que sitôt que j'aurais le comté, je saurais le gouverner ; car j'ai autant d'âme qu'un autre, et autant de corps que celui qui en a le plus, et je serais aussi roi de mon état que chacun l'est du sien; et en l'étant, je ferais ce que je voudrais; et en faisant ce que je voudrais, je ferais mon plaisir ; et en faisant mon plaisir, je serais content ; et quand on est content, on n'a plus rien à désirer ; et quand on n'a plus rien à désirer, c'est terminé, et que vienne à moi cet état, et adieu et au revoir, comme dit un aveugle à un autre.]

Notre bon écuyer utilise ici un proverbe comme locution ${ }^{23}$ conclusive. En d'autres termes, son usage ne suppose pas l'élaboration d'un raisonnement cognitif menant à une conclusion factuelle préventive ou argumentative. Il semble que Sancho considère la séquence proverbiale comme étant l'équivalent d'une locution telle que, en français : et 
voilà, et puis c'est tout ou encore et il n'y a rien à rajouter. Il ne comprend pas l'énoncé qu'il emploie et ne perçoit pas l'enseignement d'ordre moral qu'il dispense : «si on est aveugle, il est absurde de dire au revoir", soit "si notre nature ne nous permet pas de faire quelque chose, il est absurde de dire qu'on le fait ». Ce proverbe, en mettant en scène une situation absurde, a justement pour objectif de mettre en garde l'interlocuteur auquel il s'adresse contre l'absurdité de ses propres actes. Sancho s'avère être l'aveugle du proverbe qui ne saisit pas l'absurdité de ses propos ${ }^{24}$. Il nous semble envisageable de parler ici d'extra-applicabilité. En effet, le préfixe extra (mot latin signifiant étymologiquement au dehors) désignerait ce « qui est hors, qui sort du cadre, des limites de la notion exprimée par la base $»^{25}$. Cet emploi d'une séquence proverbiale à mauvais escient et la critique, même inconsciente, qu'elle génère par le truchement d'un procédé humoristique pourraient constituer une diatribe contre tous ceux qui auraient tendance à manier la matière proverbiale sans en saisir le sens. Il semble que l'on s'insurge contre ceux qui, comme c'est le cas ici, prennent les proverbes pour des locutions. Si nous nous plaçons dans une optique plus large, il serait possible de considérer que le narrateurauteur s'attaque à ceux qui parlent en toute ignorance et qui, de ce fait, sont victimes de confusions désastreuses.

Toutefois, comme nous avons déjà eu l'occasion de l'observer, le premier degré d'interprétation du Quichotte s'accompagne souvent d'un second degré, moins évident. Il semble que, dans le cas de l'absurde, les proverbes nous permettent également d'accéder à ce second niveau. Nous pensons en particulier à une réplique de Sancho (pp. 301-302) qui paraît, au premier abord, sans queue ni tête. Rappelons-en brièvement le contexte : don Quichotte s'insurge contre ceux qui ont prétendu, ou prétendent, que la reine Madásima a eu une aventure avec son médecin Elisabat. Il s'agit de personnages de romans de chevalerie :

- (...) Y de aquí tomó ocasión el vulgo ignorante y mal intencionado de decir y pensar que ella era su manceba; y mienten, digo, otra vez, y mentirán otras docientas, todos los que tal pensaren y dijeren.

- Ni yo lo digo ni lo pienso - respondió Sancho - ; allá se lo hayan : con su pan se lo coman ; si fueron amancebados, o no, a Dios habrán dado la cuenta; de mis viñas vengo, no sé nada; no soy amigo de saber vidas ajenas; que el que compra y miente, en su bolsa lo siente. Cuanto más, que desnudo nací, desnudo me hallo : ni pierdo ni gano ; mas que lo fuesen, ¿ qué me va a mí ? Y muchos piensan que hay tocinos y no hay estacas. Mas ¿ quién puede poner puertas al campo ? Cuanto más, que de Dios dijeron.

- ¡ Válame Dios - dijo don Quijote -, y qué de necedades vas, Sancho, ensartando ! i Qué va de lo que tratamos a los refranes que enhilas? [...] »

[- (...) Et de là le vulgaire ignorant et mal intentionné prit l'occasion de dire et de penser qu'elle était sa maîtresse ; mais ils mentent, je le dis, une fois encore, et ils mentiront deux cents autres fois, tous ceux qui penseront et diront telle chose.

- Moi, je ne le dis ni ne le pense - répondit Sancho - ; qu'ils se débrouillent : c'est leur affaire; s'ils furent amants, ou non, ils en auront rendu compte à Dieu; je viens de mes vignes, je ne sais rien ; je n'aime pas connaître la vie des autres; celui qui achète et ment sur le prix, sa bourse s'en ressent. D'autant plus que je suis né nu, je me trouve nu : je ne perds ni ne gagne ; mais s'ils le furent, en quoi cela me concerne-t-il moi? Et beaucoup pensent qu'il y a du lard et il n'y a même pas de pieux. Mais qui peut mettre des portes aux champs? D'autant plus qu'ils ont dit du mal de Dieu.

- Grand Dieu - dit don Quichotte -, que de sottises enfiles-tu, Sancho, les unes après les autres ! Quel est le rapport entre notre sujet et les proverbes que tu enchaînes ?] 
Sancho prononce à la suite quatre proverbes et un proverbe sous-entendu. Cette énumération semble à première vue assez disparate et incompréhensible ainsi qu'en témoigne la réaction du gentilhomme. Toutefois, dans le cas présent, si le lecteur fait un effort d'analyse, il peut réussir à comprendre cette tirade, dans un premier temps dénuée de cohérence :

- « de mis viñas vengo, no sé nada » [je viens de mes vignes, je ne sais rien] : ce proverbe est porteur d'un schéma du type «si l'on n'était pas présent, on ne peut savoir ce qui s'est passé ». Sancho veut faire entendre à son maître que dans la mesure où il n'a pas connu la reine Madásima et Elisabat, il ne peut savoir s'ils étaient ou non amants. Bref, il affirme son ignorance sur le sujet.

- « el que compra y miente, en su bolsa lo siente » [celui qui achète et ment sur le prix, sa bourse s'en ressent] : cette occurrence semble n'avoir aucun lien avec ce qui précède. Cependant, après démétaphorisation, nous obtenons le message « si on ment sur ce que l'on sait, on n'en tire aucun bénéfice ", qui implique qu'il ne faut pas mentir. L'écuyer de notre hidalgo, en énonçant ce proverbe, pourrait indiquer qu'il en respecte le principe et, par là même, sous-entendre qu'il ne ment pas au sujet de ce qu'il sait de la relation entre la reine et son médecin. Ce proverbe vient donc simplement renforcer le message de la séquence précédente.

- « desnudo nací, desnudo me hallo : ni pierdo ni gano » [je suis né nu, je me trouve nu : je ne perds ni ne gagne] : la difficulté ici pour le lecteur tient au fait que le raisonnement de Sancho s'effectue par association d'idées. En effet, le proverbe précédent faisait référence à une absence de bénéfice, voire à une perte (« en su bolsa lo siente » [sa bourse s'en ressent]). Cette idée, dans l'esprit de Panza, évoque un nouvel énoncé proverbial, qui pourrait véhiculer un schéma tel que « même si l'on est au centre de certains évènements, on ne fait pas pour autant de pertes ou de bénéfices ». Dans la logique de Sancho, « desnudo nací, desnudo me hallo : ni pierdo ni gano » revient à dire qu'il ne savait et ne sait toujours rien concernant l'affaire qui les occupe.

- «muchos piensan que hay tocinos y no hay estacas» [beaucoup pensent qu'il y a du lard et il n'y a même pas de pieux] : cette séquence signifie que « quand on pense savoir quelque chose d'important, il n'en est souvent rien » et implique une conclusion factuelle comme « il ne faut pas se fier aux apparences ». Par le biais de ce proverbe, le serviteur de don Quichotte souhaite mettre en doute la liaison de Madásima et d'Elisabat et soutenir de ce fait la vision de son maitre.

- «que de Dios dijeron » [ils ont dit du mal de Dieu] : il s'agit là du proverbe « Digan que de Dios dijeron » [Ils peuvent dire du mal de tout car ils ont dit du mal de Dieu] sous-entendu. Il vient renforcer l'idée exprimée par la phrase précédente : il est impossible de mettre des portes aux champs, soit, dans le cas présent, on ne peut empêcher la rumeur de courir, les gens de parler. Par l'intermédiaire de l'allusion proverbiale, Sancho veut indiquer au Chevalier à la Triste Figure que, puisque les mauvaises langues s'attaquent à la plus haute instance (Dieu lui-même), elles peuvent s'en prendre à tous sans que l'on n'y puisse rien.

En résumé, ce que dit Sancho, c'est qu'il ne sait rien à propos de cette histoire, qu'il ne ment pas, qu'en pareil cas l'on doit se méfier des apparences et, enfin, qu'il est impossible d'empêcher les médisances. Bref, il désire que son maitre, au vu de son ignorance et de sa franchise, ne s'en prenne pas à lui, qu'il réalise son impuissance à lutter contre les apparences trompeuses et les ragots et, en conséquence, qu'il retrouve son calme. Ce qui, de prime abord, semblait absurde ne l'est pas réellement. Avec un peu d'attention et une bonne connaissance des proverbes, le lecteur peut reconstituer le raisonnement de 
Sancho et en découvrir le sens. Du fait que les énoncés proverbiaux utilisés s'avèrent en réalité parfaitement opportuns, Sancho se révèle moins nigaud qu'il n'y paraît. Cette vision faussement absurde de l'emploi des proverbes par Panza élaborée par Cervantès semble ainsi servir le second message possible de l'œuvre. De la même façon qu'il ne faut pas se fier à l'apparence absurde de l'utilisation des proverbes par Sancho, il ne faudrait peut-être pas se limiter à voir en l'écuyer un sot et en don Quichotte un fou. Le lecteur ne devrait pas se contenter du message apparent de l'œuvre (critique des romans de chevalerie et de ceux qui confondent "réalité » et fiction) mais chercher un objectif énonciatif moins évident, plus profond auquel il ne serait possible d'accéder qu'après réflexion: les choses ne sont pas telles qu'elles le paraissent; ce ne sont pas don Quichotte et Sancho qui ont un comportement absurde, mais le reste du monde qui vit privé de toute vertu et de toute morale, et qui ne cherche plus ni à faire le bien ni à comprendre la société au sein de laquelle il évolue. La matière proverbiale, à travers son emploi en apparence absurde, concourt à dévoiler ce second degré de lecture. Ce ne sont, bien sûr, que des hypothèses tirées de nos conclusions quant à la contextualisation des proverbes dans cette œuvre et influencées par notre perception, nécessairement subjective, des choses.

L'analyse de l'utilisation de la matière proverbiale au sein de l'œuvre cervantine s'avère d'une extrême complexité parce que le Quichotte peut donner lieu à des réactions et à des interprétations divergentes mais aussi parce que le travail effectué quant au choix et à la contextualisation de chaque séquence proverbiale semble titanesque. De nombreux types d'applicabilité se croisent, se mêlent et s'entremêlent au gré de notre interprétation du message de l'œuvre si bien que le résultat obtenu semble en parfaite adéquation avec ce roman qui réunit et combine quantité de genres littéraires (roman chevaleresque, poème héroïque voire épique, roman pastoral, roman sentimental, récit autobiographique, nouvelle italienne) et de personnages. Les proverbes, à travers leurs différents usages, participent de cette volonté totalisante qui tente de rendre compte de l'ensemble des phénomènes littéraires de l'époque et des différentes facettes de la nature humaine. La matière proverbiale est également source d'enseignement, de rire mais aussi de critique puisqu'elle contribue à faire germer le doute dans l'esprit du lecteur et lui permet ainsi d'accéder à une lecture moins superficielle de l'œuvre qui pourrait mettre en évidence la désapprobation de l'auteur vis à vis de la société espagnole. La fonction d'un proverbe, stéréotype linguistique et culturel, n'est donc pas « stéréotypée », mais s'adapte à l'œuvre littéraire au sein de laquelle il est employé afin de servir le projet énonciatif de l'auteur. Comme on a coutume de le dire, l'air ne fait pas la chanson...

\section{BIBLIOGRAPHIE}

AmOSsy Ruth, HERSCHBERG PIERROT Anne, Stéréotypes et clichés, Paris, Editions Nathan, 1997.

ANSCOMBRE Jean-Claude, «La parole proverbiale », in Langage, Paris, $\mathrm{n}^{\circ}$ 139, Larousse, Septembre 2000. 
BAKHTINE Mikhaïl, Esthétique et théorie du roman, 1975, Paris, Gallimard, 2003.

BERGSON Henri, Le rire (4 ${ }^{\text {ème }}$ édition), 1899, Paris, Alcan, 1932.

BERRENDONNER Alain, Eléments de pragmatique linguistique, Paris, Les Editions de Minuit, 1981.

Boudon Pierre, « Une interface discursive : l'ironie », in Nouveaux actes sémiotiques, 49, Limoges, PULIM, 1997.

CASSIRER Ernst, Individu et cosmos dans la philosophie de la Renaissance, 1927, Paris, Les Editions de Minuit, 1983.

CERQUIGLINI Jaqueline et Bernard, «L'écriture proverbiale », in Revue des Sciences Humaines, Tome XLI, n 163, Paris, 1976.

CERVANTES SAAVEDRA Miguel de, El ingenioso hidalgo don Quijote de la Mancha, 1604, Edición de Luis Andrés Murillo, Clásicos Castalia, Madrid, 1991.

СомBEт Louis, Recherches sur le « Refranero » castillan, Paris, Société d'édition « Les Belles Lettres », 1971.

CORREAS Gonzalo, Vocabulario de refranes y frases proverbiales, 1627, Edición Louis Combet revisada por Robert Jammes y Maïté Mir-Andreu, Editorial Castalia, Madrid, 2000.

FOURNET Sonia, Etude descriptive des proverbes dans la littérature hispanique médiévale et pré-classique et de leur fonctionnement au sein des mécanismes de l'argumentation, Thèse sous la direction de Dolorès Ligatto, Limoges, 2005.

HAMON Philippe, L'ironie littéraire, Essai sur les formes de l'écriture oblique, Paris, Hachette Université, 1996.

JAUSS Hans Robert, Pour une esthétique de la réception, Paris, Gallimard, 1978.

MANDEL Oscar, «The Function of the Norme in Don Quixote » in Modern Philology, 55, Chicago, 1958.

MESCHONNIC Henri, « Les proverbes, actes de discours », in Revue des Sciences Humaines, Tome XLI, $\mathrm{n}^{\circ}$ 163, Paris, 1976.

MARAVAll José Antonio, Utopía y contrautopía en el Quijote, Santiago de Compostela, Pico Sacro, 1976.

PERRIN Laurent, «Opinion et lieu commun dans l'ironie », in Lieux Communs, topoï, stéréotypes, clichés, Christian Plantin, éd., Paris, Editions Kimé, 1993.

Postigo María Josefa, « Os provérbios de Don Quijote de la Mancha nas Traduções em Portuguès »,inRevistaVeredas, $\mathrm{N}^{\circ} 3,1999$ (http://www.geocities.com/ail_br/ osproverbiosdonquixote.htm).

RomAn Myriam, Poétique du grotesque et pratique du burlesque dans les romans hugoliens, Groupe Hugo, Université de Paris VII, 1996 (http://groupugo.div.jussieu.fr/ groupugo/96-04-06Roman.htm).

Schoentjes Pierre, Poétique de l'ironie, Paris, Seuil, 2001.

SESE Bernard \& ZUILI Marc, Vocabulaire de la langue espagnole classique (XVI è et XVIİ̀ siècles), Ouvrage dirigé par Bernard Darbord, Paris, Nathan Université, 1999.

SUARD François et BURIDANT Claude, Richesse du proverbe, Université de Lille III, P.U.L, 1984. 
VEGA Pilar, « De nuevo sobre El Quijote : novela de burlas », in Espéculo, n 11, 1999 (http:// www.ucm.es/info/especulo/numero11/vegaquij.html).

\section{NOTES}

1. Un proverbe est une " phrase figée anonyme qui exprime un avis ou un enseignement d'ordre moral ou pratique ». Sonia Fournet, Etude descriptive des proverbes dans la littérature hispanique médiévale et pré-classique et de leur fonctionnement au sein des mécanismes de l'argumentation, Thèse sous la direction de Dolorès Ligatto, Limoges, 2005, p. 12.

2. Paul-Emile Littré, Dictionnaire de la langue française, 1880, Monte-Carlo, Editions du Cap, 1966.

3. Ruth Amossy, Anne Herschberg Pierrot, Stéréotypes et clichés, Paris, Editions Nathan, 1997, p.29. Les deux auteurs citent Jahoda, Morfaux et Fischer comme détracteurs du stéréotype.

4. Id.

5. Henri Meschonnic, «Les proverbes, actes de discours », in Revue des Sciences Humaines, Tome XLI, n 163, Paris, 1976, p. 421.

6. Miguel de Cervantes Saavedra, El ingenioso hidalgo don Quijote de la Mancha, 1604, Edición de Luis Andrés Murillo, Clásicos Castalia, Madrid, 1991. Pour nous référer à l'oeuvre et au personnage, nous emploierons à présent le terme français "Quichotte", traduction attestée de l'espagnol "Quijote".

7. En effet, l'ami anonyme de l'auteur donne son opinion sur le Quichotte en ces termes : «[...] si bien caigo en la cuenta, este vuestro libro no tiene necesidad de ninguna cosa de aquellas que vos decís que le falta, porque todo él es una invectiva contra los libros de cavallerías [...] » (p. 57).

8. S. Fournet, op. cit., p. 282. L'applicabilité se distingue de la notion d'appropriété élaborée par Moeschler dans la mesure où cette dernière se limite à évaluer le respect des contraintes d'enchaînement (cohérence/cohésion) des séquences conversationnelles et ne concerne donc que la formation (bonne ou mauvaise) des unités dialogiques de la conversation.

9. « L'œuvre littéraire n'est pas un objet existant en soi et qui présenterait en tout temps à tout observateur la même apparence. [...] Elle est plutôt faite [...] pour éveiller à chaque lecture une résonance nouvelle qui arrache le texte à la matérialité des mots et actualise son existence [...]. » Hans Robert Jauss, Pour une esthétique de la réception, Paris, Gallimard, Bibliothèque des idées, 1978, p. 47.

10. L'ironie consisterait à exprimer une opinion tout en laissant suffisamment d'indices implicites impliquant que l'on désapprouve cette opinion et que l'on soutient une opinion, non pas nécessairement contraire, mais différente. Il s'agit donc d'un phénomène permettant d'exprimer sa désapprobation vis à vis d'un fait, d'une attitude. L'ironie que nous pourrions observer ici lors de l'emploi des proverbes ne serait pas le fait du personnage énonciateur de la séquence proverbiale mais de l'auteur, qui utiliserait des énoncés proverbiaux non applicables au contexte (personnages, situation d'énonciation). 11. Par souci de clarté, nous proposons une traduction des proverbes employés ainsi que du discours au sein duquel ils sont incorporés. Nous avons préféré transcrire le plus littéralement possible les énoncés étudiés dans la mesure où il nous semble que la moelle d'un proverbe ne peut être rendue qu'en essayant de rester conforme à la lettre, au mot à 
mot du texte. Ce choix risque d'occasionner des lourdeurs mais a l'avantage de rester au plus près du sens et de l'imagerie employée.

12. L'œuvre de Cervantès a toujours été et continue d'être l'objet d'interprétations multiples et variées susceptibles de se scinder en deux tendances générales que 0 . Mandel (Oscar Mandel, «The Function of the Norme in Don Quixote », in Modern Philology, 55, Chicago, 1958) a qualifiées de « hard » et de " soft ». La première considère le Quichotte comme une satire, une parodie des romans de chevalerie où l'hidalgo de la Manche n'est qu'un fou ridicule. La possibilité d'une seconde lecture qui ferait du Chevalier à la Triste Figure un héros admirable a été clairement pressentie dès 1789 par Cadalso qui écrit, dans ses Cartas Marruecas, à propos de l'ouvrage en question : « En esta nación hay un libro muy aplaudido por todas las demás. Lo he leído, y me ha gustado sin duda ; pero no deja de mortificarme la sospecha de que el sentido literal es uno, y el verdadero es otro muy diferente. » (José Cadalso, Cartas Marruecas, 1789, Madrid, Espasa Calpe, 1971, Carta LXI). Dès lors, les interprétations cherchant à découvrir le « véritable » message du Quichotte n'ont cessé de croître.

13. Aux dires de J. A. Maravall, Cervantès proposerait en effet « la fábula de un caballero imposible, para que su misma imposibilidad dé cuenta de la penosa situación de una sociedad en la que no tienen sentido los nobles propósitos que al hilo de la invención novelesca se van ofreciendo ». (José Antonio Maravall, Utopía y contrautopía en el Quijote, Santiago de Compostela, Pico Sacro, 1976, p. 245)

14. L'humour serait une forme d'esprit railleuse qui attire l'attention, avec détachement, sur les aspects plaisants ou insolites de la réalité.

15. Comme le souligne Myriam Roman, il naît généralement « de l'écart entre un sujet et un style, entre noblesse et bassesse. [...] Pour parler de burlesque, il faut d'une part pouvoir identifier une opposition entre le style et le sujet, d'autre part que le style "bas" l'emporte et mette à mal la "noblesse du sujet" ». Myriam Roman, Poétique du grotesque et pratique du burlesque dans les romans hugoliens, Groupe Hugo, Université de Paris VII, 1996 (http://groupugo.div.jussieu.fr/groupugo/96-04-06Roman.htm).

16. Une telle interprétation, en dévoilant un double sens du vocable " diablo ", supposerait une bi-applicabilité également involontaire du proverbe qui conduirait à l'apparition d'un portrait peu flatteur des deux protagonistes concernés. La signification de « diablo » serait ainsi fonction du sens que l'on donnerait à « olor ».

17. Un proverbe d'expression indirect exprime figurativement un enseignement ou un avis d'ordre moral ou pratique.

18. Le Trésor de la Langue Française informatisé, 2002 (http://atilf.atilf.fr/tlfv3.htm).

19. Gonzalo Correas, Vocabulario de refranes y frases proverbiales, 1627, Edición Louis Combet revisada por Robert Jammes y Maïté Mir-Andreu, Editorial Castalia, Madrid, 2000. Juan Bergua, Refranero español, Madrid, Ediciones Ibéricas, 1992. Jorge Castillo, Refranero español, Barcelona, Edicomunicación, 1999.

20. L'allusion à un proverbe dont on force l'applicabilité produit un effet humoristique dû à la rupture de la norme.

21. Cette volonté de singulariser le personnage de don Quichotte pourrait aller de pair avec l'émergence du sujet (conscience de soi) en philosophie. La Renaissance (XVè-XVI ${ }^{\grave{a}}$ siècles) fut, en effet, marquée par une reprise du débat sur l'individu et la liberté. Selon $E$. Cassirer, l'origine de l'individualisme moderne remonte aux Quattrocento et Cinquecento avec les réflexions de Nicolas de Cues, Pic de la Mirandole, Ficin, Charles de Bovelles et Paracelse. Ces penseurs ont renoué avec une problématique qui était celle de Socrate et de Platon, premiers à développer "l'intuition du moi" et à affirmer l'âme comme principe 
de la subjectivité. Leurs travaux ont engendré une nouvelle conception de la conscience de soi, préparant ainsi une rupture progressive avec la logique scolastique et présentant une conception moderne de la religion qui annonce la Réforme. Ernst Cassirer, Individu et cosmos dans la philosophie de la renaissance, 1927, Paris, Les Editions de Minuit, 1983.

22. « [L]e sentiment de l'absurdité ne naît pas du simple examen d'un fait ou d'une impression mais [...] jaillit de la comparaison entre un état de fait et une certaine réalité, entre une action et le monde qui la dépasse. L'absurde est essentiellement un divorce. Il n'est ni dans l'un ni dans l'autre des éléments comparés. Il naît de leur confrontation. » Albert Camus, Le mythe de Sisyphe, 1942, Paris, Gallimard, 1996, p. 50.

23. Par locution, nous désignons toute combinaison figée ou semi-figée de deux termes ou plus ayant une fonction autre que didactique. S. Fournet, Etude descriptive..., op. cit., p. 16. 24. L'emploi absurde de l'énoncé se double ici d'un emploi humoristique ayant pour origine une bi-applicabilité : la pluralité sémantique du terme « ciego » - aveugle - (qui est privé de l'usage de la vue vs qui manque de discernement) vise à se rire du personnage auquel le proverbe se réfère analogiquement, à savoir, dans le cas présent, son propre locuteur. Il pourrait donc s'agir d'une autocritique involontaire qui mettrait justement en évidence l'usage absurde que fait Sancho du proverbe.

25. Le Trésor de la langue française..., op. cit.

\section{RÉSUMÉS}

Le «langage stéréotypique » représente généralement l'ensemble des structures de la langue mettant en avant le savoir partagé et la connaissance du monde d'une société linguistique. Or, les proverbes ont de tous temps constitué la sagesse des nations ou sagesse populaire. La matière proverbiale représente donc, pour reprendre les mots de Henri Meschonnic, " une tentative empirique de mettre le monde en ordre ». Nous rejoignons ici la fonction fondamentale du stéréotype grâce à laquelle chaque individu a la possibilité de régler ses actions. Notre objectif est de tenter de démontrer que les proverbes, en tant que stéréotypes - à la fois linguistiques et culturels -, ne sont pas de simples ornements mais ont pour fonction de servir, en discours, la stratégie énonciative de l'œuvre littéraire au sein de laquelle ils sont incorporés. Nous avons choisi comme terrain d'analyse le Don Quijote de la Mancha de Miguel de Cervantès.

El "lenguaje estereotípico" suele representar el conjunto de las estructuras de la lengua que ponen de realce el saber compartido y el conocimiento del mundo de una sociedad linguística. Ahora bien, los refranes siempre constituyeron la sabiduría de las naciones o sabiduría popular. Dichos enunciados representan, pues, según Henri Meschonnic, "une tentative empirique de mettre le monde en ordre". Coincidimos en este punto con la función básica del estereotipo mediante la cual cada individuo puede ajustar sus acciones. Nuestra meta es intentar demostrar que los refranes, como esteretipos - linguísticos y culturales a la vez -, no son meros adornos sino que tienen como función servir, en discurso, la estrategia enunciativa de la obra literaria a la cual se incorporan. Hemos elegido como corpus el Don Quijote de la Mancha de Miguel de Cervantes. 
INDEX

Mots-clés : proverbe, applicabilité, ironie, humour, absurde, littérature pré-classique espagnole, stratégie énonciative

\section{AUTEUR}

SONIA FOURNET-PEROT

Maître de conférences d'espagnol à l'Université de Limoges, CERES 